\title{
Kantorovich-Rubinstein Maximum Principle in the Stability Theory of Markov Semigroups
}

by

\author{
Henryk GACKI \\ Presented by Andrzej LASOTA
}

Summary. A new sufficient condition for the asymptotic stability of a locally Lipschitzian Markov semigroup acting on the space of signed measures $\mathcal{M}_{\text {sig }}$ is proved. This criterion is applied to the semigroup of Markov operators generated by a Poisson driven stochastic differential equation.

1. Introduction. The purpose of the present paper is to show an application of the Kantorovich-Rubinstein maximum principle in the stability theory of Markov semigroups. This principle was already used to prove the stability of some iterated function systems, stochastically perturbed dynamical systems with discrete time (see [1]) and stochastic semigroups generated by the Tjon-Wu equation (see [8]).

We will also show the application of the general theory to the semigroup $\left(P^{t}\right)_{t \geq 0}$ of Markov operators generated by a Poisson driven stochastic differential equation. This equation has the form

$$
d \xi(t)=a(\xi(t)) d t+\int_{\Theta} \sigma(\xi(t), \theta) \mathcal{N}_{p}(d t, d \theta) \quad \text { for } t \geq 0
$$

and will be considered with the initial condition

$$
\xi(0)=\xi^{0},
$$

where $\{\xi(t)\}_{t>0}$ is a stochastic process with values in the $d$-dimensional real space $\mathbb{R}^{d}$. In the special case $\xi(0)=x$ a.s. this solution will be denoted by $\xi_{x}$.

2000 Mathematics Subject Classification: Primary 60H10; Secondary 37J25.

Key words and phrases: asymptotic stability, Poisson driven stochastic equation, Markov semigroup. 
It is known that for sufficiently regular $f$ and under some regularity conditions concerning the coefficients, the function

$$
u(t, x)=E f\left(\xi_{x}(t)\right),
$$

where $E$ denotes the mathematical expectation, satisfies the equation

$$
u_{t}(t, x)=\left\langle a(x) \mid u_{x}(t, x)\right\rangle-\lambda u(t, x)+\lambda \int_{\Theta} u(t, x+\sigma(x, \theta)) \widetilde{n}(d \theta),
$$

where

$$
u(0, x)=f(x) \quad \text { for } x \in \mathbb{R}^{d} .
$$

The semigroup $\left(U^{t}\right)_{t \geq 0}$ is defined by the formula

$$
U^{t} f(x)=u(t, x) \quad \text { for } t \geq 0, x \in \mathbb{R}^{d}
$$

and the semigroup $\left(P^{t}\right)_{t \geq 0}$ is adjoint to $\left(U^{t}\right)_{t \geq 0}$. The precise conditions concerning equation (1) and the formal definitions of the semigroup $\left(P^{t}\right)_{t \geq 0}$ and $\left(U^{t}\right)_{t \geq 0}$ will be given in Section 4 .

The asymptotic properties of the semigroup $\left(P^{t}\right)_{t \geq 0}$ generated by the stochastic differential equation (1) were studied in $[6,10,12]$.

In particular J. Malczak [10] found a criterion of asymptotic stability in the case when the operators $P^{t}$ are defined on the space $L^{1}\left(\mathbb{R}^{d}\right)$. Using a double contraction principle A. Lasota [6] proved the asymptotic stability for $P^{t}$ acting on the space of signed measures. Those results were generalized by J. Traple [12] who considered equation (1) in a quite general situation when the intensity $\lambda$ of the Poisson process depends on the solution.

Our result intersects with that of Traple. We assume that $\lambda$ is constant but our conditions on the coefficients are less restrictive.

2. Preliminaries. Let $(X, \varrho)$ be a metric space. We will assume that every closed and bounded subset of $X$ is compact. This condition implies that $(X, \varrho)$ is a Polish space.

We denote by $\mathcal{B}(X)$ the $\sigma$-algebra of Borel subsets of $X$ and by $\mathcal{M}$ the family of all finite Borel measures (nonnegative, $\sigma$-additive) on $X$.

Let $\mathcal{M}_{1}$ denote the subset of those $\mu \in \mathcal{M}$ for which $\mu(X)=1$. The elements of $\mathcal{M}_{1}$ will be called distributions. Further let

$$
\mathcal{M}_{\text {sig }}=\left\{\mu_{1}-\mu_{2}: \mu_{1}, \mu_{2} \in \mathcal{M}\right\}
$$

be the space of finite signed measures.

Let $c$ be a fixed element of $X$. For every $\alpha \geq 1$ we define the subset $\mathcal{M}_{1, \alpha}$ of $\mathcal{M}_{1}$ by setting

$$
\mathcal{M}_{1, \alpha}=\left\{\mu \in \mathcal{M}_{1}: \int_{X}(\varrho(x, c))^{\alpha} \mu(d x)<\infty\right\} .
$$

It is evident that this space does not depend on the choice of $c$. 
As usual, $B(X)$ denotes the space of all bounded Borel measurable functions $f: X \rightarrow \mathbb{R}$, and $C_{\mathrm{b}}(X)$ the subspace of all bounded continuous functions, both endowed with the supremum norm

$$
\|f\|=\sup _{x \in X}|f(x)| .
$$

For every $f: X \rightarrow \mathbb{R}$ and $\mu \in \mathcal{M}_{\text {sig }}$ we write

$$
\langle f, \mu\rangle=\int_{X} f(x) \mu(d x),
$$

whenever this integral exists.

In the space $\mathcal{M}_{1}$ we introduce the Hutchinson metric (see [2]) by

$$
\left\|\mu_{1}-\mu_{2}\right\|_{\mathcal{H}}=\sup \left\{\left|\left\langle f, \mu_{1}-\mu_{2}\right\rangle\right|: f \in \mathcal{H}\right\},
$$

where $\mathcal{H}$ is the set of functions $f: X \rightarrow \mathbb{R}$ which satisfy

$$
|f(x)-f(y)| \leq \varrho(x, y) \quad \text { for } x, y \in X .
$$

The value $\left\|\mu_{1}-\mu_{2}\right\|_{\mathcal{H}}$ in (4) is well defined and finite for $\mu_{1}, \mu_{2} \in \mathcal{M}_{1,1}$.

Furthermore, for $\mu_{1}, \mu_{2} \in \mathcal{M}_{1}$, we have

$$
\left\|\mu_{1}-\mu_{2}\right\|_{\mathcal{H}}=\sup \left\{\left|\left\langle f, \mu_{1}-\mu_{2}\right\rangle\right|: f \in \mathcal{H}_{c}\right\},
$$

where $c$ is a fixed element of $X$ and $\mathcal{H}_{c}=\{f \in \mathcal{H}: f(c)=0\}$.

In $\mathcal{M}_{1}$ we also introduce the Fortet-Mourier metric by setting

$$
\left\|\mu_{1}-\mu_{2}\right\|_{\mathcal{F}}=\sup \left\{\left|\left\langle f, \mu_{1}-\mu_{2}\right\rangle\right|: f \in \mathcal{F}\right\},
$$

where $\mathcal{F}$ is the set of functions $f: X \rightarrow \mathbb{R}$ satisfying

$$
\|f\| \leq 1, \quad|f(x)-f(y)| \leq \varrho(x, y) \quad \text { for } x, y \in X .
$$

We say that a sequence $\left(\mu_{n}\right) \subset \mathcal{M}_{1}$ converges weakly to a measure $\mu \in$ $\mathcal{M}_{1}$ if

$$
\lim _{n \rightarrow \infty}\left\langle f, \mu_{n}\right\rangle=\langle f, \mu\rangle \quad \text { for } f \in C_{\mathrm{b}}(X) .
$$

Since $X$ is a Polish space, condition (8) is equivalent to

$$
\lim _{n \rightarrow \infty}\left\|\mu_{n}-\mu\right\|_{\mathcal{F}}=0 .
$$

Moreover $\mathcal{M}_{1}$ with the distance $\left\|\mu_{n}-\mu\right\|_{\mathcal{F}}$ is a complete metric space (see [11]).

Denote by $K(x, r)$ the closed ball in $X$ with center $x \in X$ and radius $r$. Let $\mu \in \mathcal{M}_{1}$. We define the support of $\mu$ by setting

$$
\operatorname{supp} \mu=\{x \in X: \mu(K(x, \varepsilon))>0 \text { for every } \varepsilon>0\} .
$$

REMARK 1. Every set $\mathcal{M}_{1, \alpha}$ for $\alpha \geq 1$ contains the subset of all measures $\mu \in \mathcal{M}_{1}$ with compact support. This subset is dense in $\mathcal{M}_{1}$ with respect to the Fortet-Mourier norm. Thus for every $\alpha \geq 1$ the set $\mathcal{M}_{1, \alpha}$ is dense in $\mathcal{M}_{1}$ with respect to that norm. 
3. Markov semigroups. A linear mapping $P: \mathcal{M}_{\text {sig }} \rightarrow \mathcal{M}_{\text {sig }}$ is called a Markov operator if $P\left(\mathcal{M}_{1}\right) \subset \mathcal{M}_{1}$.

Remark 2. Every Markov operator is defined by its values on $\mathcal{M}_{1}$.

A Markov operator $P$ is called a Feller operator if there is a linear operator $U: C_{\mathrm{b}}(X) \rightarrow C_{\mathrm{b}}(X)$, dual to $P$, i.e.,

$$
\langle U f, \mu\rangle=\langle f, P \mu\rangle \quad \text { for } f \in C_{\mathrm{b}}(X), \mu \in \mathcal{M}_{1} .
$$

REMARK 3. The dual operator $U$ has a unique extension to the set of all Borel measurable, nonnegative, not necessarily bounded functions on $X$. The extension is given by

$$
(U f)(x)=\left\langle f, P \delta_{x}\right\rangle,
$$

where $\delta_{x} \in \mathcal{M}_{1}$ is the Dirac measure supported at $x$.

A Markov operator $P$ is called Lipschitzian with a constant $k>0$ if

$$
\left\|P \mu_{1}-P \mu_{2}\right\|_{\mathcal{F}} \leq k\left\|\mu_{1}-\mu_{2}\right\|_{\mathcal{F}} \quad \text { for } \mu_{1}, \mu_{2} \in \mathcal{M}_{1} .
$$

If $k \leq 1$ then $P$ is nonexpansive.

A family of Markov operators $\left(P^{t}\right)_{t \geq 0}$ is called a semigroup if

$$
P^{t+s}=P^{t} P^{s} \quad \text { for } t, s \in \mathbb{R}^{+}
$$

and $P^{0}=I$ is the identity operator on $\mathcal{M}_{\text {sig. }}$.

If the operators $P^{t}, t \geq 0$, are Fellerian, we say that $\left(P^{t}\right)_{t \geq 0}$ is a Feller semigroup. We denote by $\left(U^{t}\right)_{t \geq 0}$ the semigroup dual to $\left(P^{t}\right)_{t \geq 0}$.

We say that a semigroup $\left(P^{t}\right)_{t \geq 0}$ is weakly continuous if $P^{t} \mu \rightarrow \mu$ weakly as $t \rightarrow 0^{+}$, for every $\mu \in \mathcal{M}_{1}$.

A Markov semigroup $\left(P^{t}\right)_{t \geq 0}$ is called locally Lipschitzian if there exists a locally bounded function $k: \mathbb{R}^{+} \rightarrow \mathbb{R}^{+}$such that for every $t \geq 0$ the operator $P^{t}$ is Lipschitzian with constant $k(t)$; if $k(t) \leq 1$ for all $t \in \mathbb{R}^{+}$, then $\left(P^{t}\right)_{t \geq 0}$ is a nonexpansive semigroup.

A nonexpansive semigroup $\left(P^{t}\right)_{t \geq 0}$ is called strongly contracting on $\mathcal{M}_{1, \alpha}$ if for any $\mu_{1}, \mu_{2} \in \mathcal{M}_{1, \alpha}, \mu_{1} \neq \mu_{2}$, there is a $t_{0} \in \mathbb{R}^{+}$such that

$$
\left\|P^{t_{0}} \mu_{1}-P^{t_{0}} \mu_{2}\right\|_{\mathcal{H}}<\left\|\mu_{1}-\mu_{2}\right\|_{\mathcal{H}} \text {. }
$$

A measure $\mu_{*} \in \mathcal{M}$ is called stationary (or invariant) for a Markov semigroup $\left(P^{t}\right)_{t \geq 0}$ if

$$
P^{t} \mu_{*}=\mu_{*} \quad \text { for } t \geq 0 .
$$

A Markov semigroup $\left(P^{t}\right)_{t \geq 0}$ is called asymptotically stable if there is a stationary distribution $\mu_{*}$ such that

$$
\lim _{t \rightarrow \infty}\left\|P^{t} \mu-\mu_{*}\right\|_{\mathcal{F}}=0 \quad \text { for all } \mu \in \mathcal{M}_{1} .
$$

The distribution $\mu_{*}$ satisfying (12) is unique. 
In the proof of our main result Theorem 1 we will use the following properties of the Hutchinson norm:

Kantorovich-Rubinstein Maximum Principle. For any $\mu_{1}, \mu_{2} \in$ $\mathcal{M}_{1,1}, \mu_{1} \neq \mu_{2}$, there exists $f \in \mathcal{H}$ such that

$$
\left\langle f, \mu_{1}-\mu_{2}\right\rangle=\left\|\mu_{1}-\mu_{2}\right\|_{\mathcal{H}} .
$$

Moreover, every such $f$ satisfies the condition

$$
|f(x)-f(y)|=\varrho(x, y)
$$

for some $x, y \in X, x \neq y$.

(For details see [11, pp. 89-121]).

Let $c$ be a fixed element of $X$ and $\varrho_{c}^{\alpha}(x):=\varrho^{\alpha}(x, c)$ for every $\alpha>0$.

Theorem 1. Let $\left(P^{t}\right)_{t \geq 0}$ be a Feller semigroup and $\left(U^{t}\right)_{t \geq 0}$ its dual semigroup. Assume that there is $t_{0} \in \mathbb{R}^{+}$such that for every $f \in \mathcal{H}$ the following two conditions are satisfied:

$$
\begin{aligned}
\left|U^{t} f(x)-U^{t} f(y)\right| & \leq \varrho(x, y) \quad \text { for } x, y \in X \text { and } t \geq 0, \\
\left|U^{t_{0}} f(x)-U^{t_{0}} f(y)\right| & <\varrho(x, y) \quad \text { for } x, y \in X, x \neq y .
\end{aligned}
$$

Moreover, assume that there exist constants $A, B \geq 0$ and $\alpha>1$ such that

$$
\left(U^{t} \varrho_{c}^{\alpha}\right)(x) \leq A \varrho_{c}^{\alpha}(x)+B \quad \text { for } x \in X \text { and } t \geq 0 .
$$

Then $\left(P^{t}\right)_{t \geq 0}$ is asymptotically stable.

Proof. From (16), it follows immediately that

$$
P^{t}\left(\mathcal{M}_{1, \alpha}\right) \subset \mathcal{M}_{1, \alpha} \quad \text { for } t \geq 0 .
$$

Moreover, by (14)-(16) the Feller semigroup $\left(P^{t}\right)_{t \geq t_{0}}$ is nonexpansive on $\mathcal{M}_{1, \alpha}$ with respect to the Hutchinson norm. Indeed, for $\mu_{1}, \mu_{2} \in \mathcal{M}_{1, \alpha}$ and $t \geq 0$ we have

$$
\begin{aligned}
\left\|P^{t} \mu_{1}-P^{t} \mu_{2}\right\|_{\mathcal{H}} & =\sup \left\{\left|\left\langle f, P^{t} \mu_{1}-P^{t} \mu_{2}\right\rangle\right|: f \in \mathcal{H}\right\} \\
& =\sup \left\{\left|\left\langle U^{t} f, \mu_{1}-\mu_{2}\right\rangle\right|: f \in \mathcal{H}\right\} \leq\left\|\mu_{1}-\mu_{2}\right\|_{\mathcal{H}} .
\end{aligned}
$$

We claim that $\left(P^{t}\right)_{t \geq 0}$ is also strongly contracting with respect to the same norm, i.e.,

$$
\left\|P^{t_{0}} \mu_{1}-P^{t_{0}} \mu_{2}\right\|_{\mathcal{H}}<\left\|\mu_{1}-\mu_{2}\right\|_{\mathcal{H}} \quad \text { for } \mu_{1}, \mu_{2} \in \mathcal{M}_{1, \alpha}, \mu_{1} \neq \mu_{2} .
$$

Fix $\mu_{1}, \mu_{2} \in \mathcal{M}_{1, \alpha}, \mu_{1} \neq \mu_{2}$. According to the Kantorovich-Rubinstein maximum principle there exists $f \in \mathcal{H}$ such that

$$
\left\langle f, P^{t_{0}} \mu_{1}-P^{t_{0}} \mu_{2}\right\rangle=\left\|P^{t_{0}} \mu_{1}-P^{t_{0}} \mu_{2}\right\|_{\mathcal{H}} .
$$

This equality may be rewritten in the form

$$
\left\langle U^{t_{0}} f, \mu_{1}-\mu_{2}\right\rangle=\left\|P^{t_{0}} \mu_{1}-P^{t_{0}} \mu_{2}\right\|_{\mathcal{H}} .
$$


The function $U^{t_{0}} f$ satisfies (15), so according to the second part of the principle we obtain

$$
\left\langle U^{t_{0}} f, \mu_{1}-\mu_{2}\right\rangle<\left\|\mu_{1}-\mu_{2}\right\|_{\mathcal{H}} .
$$

Conditions (18) and (19) imply (17) and the claim is proved.

Now we are going to verify that for every $\mu \in \mathcal{M}_{1, \alpha}$ the trajectory $\left\{P^{t} \mu\right\}_{t \geq 0}$ is relatively compact in $\mathcal{M}_{1, \alpha}$. Let $\left(t_{n}\right)$ be a sequence of integers such that $t_{n} \rightarrow \infty$ and $t_{n} \geq 0$ for $n=1,2, \ldots$.

According to (16) the sequence $\left(P^{t_{n}} \mu\right)$ satisfies the Prokhorov condition (see $[8$, p. 66]). So from the Prokhorov theorem it follows immediately that there exists a subsequence $\left(P^{t_{k_{n}}} \mu\right)$ which converges weakly to a measure $\mu_{0} \in \mathcal{M}_{1}$. Now we are going to show that $\mu_{0} \in \mathcal{M}_{1, \alpha}$ and $\left(P^{t_{k_{n}}} \mu\right)$ is convergent to $\mu_{0}$ in the Hutchinson norm. For given $r>0$ define

$$
g_{r}(x)= \begin{cases}\varrho_{c}^{\alpha}(x) & \text { for } x \in K(c, r), \\ r^{\alpha} & \text { for } x \notin K(c, r) .\end{cases}
$$

Condition (16) implies that

$$
\left\langle g_{r}, P^{t_{k_{n}}} \mu\right\rangle=\left\langle U^{t_{k_{n}}} g_{r}, \mu\right\rangle \leq l, \quad \text { where } \quad l=A\left\langle\varrho_{c}^{\alpha}, \mu\right\rangle+B .
$$

The function $g_{r}$ is continuous and bounded. Consequently,

$$
\lim _{n \rightarrow \infty}\left\langle g_{r}, P^{t_{k_{n}}} \mu\right\rangle=\left\langle g_{r}, \mu_{0}\right\rangle .
$$

Since $r>0$ was arbitrary, the last equality and (20) imply that $\mu_{0} \in \mathcal{M}_{1, \alpha}$. So it suffices to verify that

$$
\lim _{n \rightarrow \infty}\left\|P^{t_{k_{n}}} \mu-\mu_{0}\right\|_{\mathcal{H}}=0 .
$$

An elementary calculation shows that

$$
\int_{X \backslash K(c, r)} \varrho_{c}(x) P^{t_{k_{n}}} \mu(d x) \leq \frac{l}{r^{\alpha-1}} .
$$

Fix an $\varepsilon>0$ and choose $r$ such that $4 l / r^{\alpha-1} \leq \varepsilon$. Define

$$
\Delta=[-r, r], \quad \mathcal{F}_{\Delta, 1}=\{f \in C(X):|f(x)| \leq r \text { and }|f(x)-f(y)| \leq \varrho(x, y)\} .
$$

On $\mathcal{M}_{1}$ the metric

$$
\left\|\mu_{1}-\mu_{2}\right\|_{\mathcal{F}_{\Delta, 1}}=\sup \left\{\left\langle f, \mu_{1}-\mu_{2}\right\rangle: f \in \mathcal{F}_{\Delta, 1}\right\},
$$

is equivalent to the Fortet-Mourier metric. For $f \in \mathcal{H}$ define

$$
f_{r}(x)=\max \{\min [f(x), r],-r\} .
$$

Evidently $f_{r} \in \mathcal{F}_{\Delta, 1}$. Furthermore for $f \in \mathcal{H}_{c}$ the function $f_{r}$ has the following properties:

(a) $f_{r}(x)=f(x)$ for $x \in K(r, c)$,

(b) $\left|f(x)-f_{r}(x)\right| \leq 2 \varrho_{c}(x)$ for $x \in X$. 
From this and (21), it follows immediately that

$$
\left\langle f, P^{t_{k_{n}}} \mu-\mu_{0}\right\rangle \leq\left\|P^{t_{k_{n}}} \mu-\mu_{0}\right\|_{\mathcal{F}_{\Delta, 1}}+\frac{4 l}{r^{\alpha-1}} \leq\left\|P^{t_{k_{n}}} \mu-\mu_{0}\right\|_{\mathcal{F}_{\Delta, 1}}+\varepsilon
$$

for $f \in \mathcal{H}_{c}$. This shows that $\left(P^{t_{k_{n}}} \mu\right)$ converges to $\mu_{0}$ in the Hutchinson norm. Thus the trajectory $\left\{P^{t} \mu\right\}_{t \geq 0}$ is compact in $\mathcal{M}_{1, \alpha}$.

Condition (17) and the compactness of orbits imply that for every $t_{n} \rightarrow \infty$ the sequence $\left(P^{t_{n}} \mu\right)$ with $\mu \in \mathcal{M}_{1, \alpha}$ converges in the Hutchinson norm to a unique invariant measure $\mu_{0}$ (see [5, p. 115]).

To complete the proof it is sufficient to observe that $\mathcal{M}_{1, \alpha}$ is dense in $\mathcal{M}_{1}$ and by (14), (15) the Feller semigroup $\left(P^{t}\right)_{t \geq 0}$ is nonexpansive on $\mathcal{M}_{1}$ with respect to the Fortet-Mourier norm.

The analogue of Theorem 1 for locally Lipschitzian Markov semigroups is

TheOREM 2. Let $\left(P^{t}\right)_{t \geq 0}$ be a locally Lipschitzian Markov semigroup on $\mathcal{M}_{\text {sig }}$ and let $\left(U^{t}\right)_{t \geq 0}$ denote the semigroup dual to $\left(P^{t}\right)_{t \geq 0}$. Assume that there is $t_{0} \in \mathbb{R}^{+}$such that

$$
\left|U^{t_{0}} f(x)-U^{t_{0}} f(y)\right|<\varrho(x, y) \quad \text { for } x, y \in X, x \neq y,
$$

for every $f \in \mathcal{H}$. Moreover, assume that there exist constants $A, B \geq 0$ and $\alpha>1$ such that

$$
\left(U^{n t_{0}} \varrho_{c}^{\alpha}\right)(x) \leq A \varrho_{c}^{\alpha}(x)+B \quad \text { for } x \in X \text { and } n=0,1,2, \ldots
$$

Then $\left(P^{t}\right)_{t \geq 0}$ is asymptotically stable.

The proof can be easily derived from an analogous result in [1].

4. Application. In this part we denote by $\|\cdot\|,\langle\cdot \mid \cdot\rangle$ the Euclidean norm and the Euclidean scalar product in $\mathbb{R}^{d}$. The spaces

$$
B_{\mathrm{b}}\left(\mathbb{R}^{d}\right) \supset C_{\mathrm{b}}\left(\mathbb{R}^{d}\right) \supset C_{\mathrm{c}}^{1}\left(\mathbb{R}^{d}\right)
$$

are respectively: the space of all real, Borel measurable, bounded functions on $\mathbb{R}^{d}$ equipped with the supremum norm; the subspace of all bounded continuous functions; the subspace of all functions with compact support and continuous first derivatives.

In our study of solutions of (1), (2) we make the following assumptions:

(i) The coefficient $a: \mathbb{R}^{d} \rightarrow \mathbb{R}^{d}$ is Lipschitzian,

$$
\|a(x)-a(y)\| \leq l_{a}\|x-y\| \quad \text { for } x, y \in \mathbb{R}^{d} .
$$

(ii) $(\Theta, \mathcal{G}, \widetilde{n})$ is a finite measure space with $\widetilde{n}(\Theta)=1$.

(iii) The perturbation coefficient $\sigma: \mathbb{R}^{d} \times \Theta \rightarrow \mathbb{R}^{d}$ is a $\mathcal{B}_{\mathbb{R}^{d}} \times \mathcal{G} / \mathcal{B}_{\mathbb{R}^{d^{-}}}$ measurable function such that

$$
|\sigma(x, \cdot)-\sigma(y, \cdot)|_{L^{2}(\widetilde{n})} \leq l_{\sigma}\|x-y\| \quad \text { for } x, y \in \mathbb{R}^{d} .
$$


(iv) The mapping $q: \mathbb{R}^{d} \times \Theta \rightarrow \mathbb{R}^{d}$ given by

$$
q(z, \theta)=z+\sigma(z, \theta) \quad \text { for } z \in \mathbb{R}^{d}, \theta \in \Theta
$$

is measurable and $q(x, \cdot) \in L^{1}(\widetilde{n})$ for $x \in \mathbb{R}^{d}$. Moreover,

$$
|q(x, \cdot)-q(y, \cdot)|_{L^{1}(\widetilde{n})} \leq l_{q}\|x-y\| \quad \text { for } x, y \in \mathbb{R}^{d}
$$

for some constant $l_{q} \geq 0$.

(v) There is a probability space $(\Omega, \mathcal{F}$, prob), a sequence of nonnegative random variables $\left(t_{i}\right)_{i \in \mathbb{N}_{0}}$ and a sequence of random elements $\left(\theta_{i}\right)_{i \in \mathbb{N}}$ with values in $\Theta$. The variables $\Delta t_{i}=t_{i}-t_{i-1}\left(t_{0}=0\right)$ are nonnegative, independent and equally distributed with probability density function $\lambda e^{-\lambda t}$ for $t \geq 0$. The elements $\theta_{i}$ are independent, equally distributed with distribution $\widetilde{n}$. The sequences $\left(t_{i}\right)$ and $\left(\theta_{i}\right)$ are also independent. Under this condition the mapping

$$
\Omega \ni \omega \mapsto p(\omega)=\left(t_{i}(\omega), \theta_{i}(\omega)\right)_{i \in \mathbb{N}}
$$

defines a stationary Poisson point process ([3, Ch. 1, p. 44]).

(vi) For every $\mu \in \mathcal{M}_{1}$ there is an $\mathbb{R}^{d}$-valued random vector $\xi_{\mu}$ defined on $\Omega$, independent of $p$ and having distribution $\mu$.

Condition (v) implies that for every measurable set $Z \subset(0, \infty) \times \Theta$ the variable

$$
\mathcal{N}_{p}(Z)=\#\left\{i:\left(t_{i}, \theta_{i}\right) \in Z\right\}
$$

is Poisson distributed. It is called the Poisson random counting measure.

Denote by $E$ the mathematical expectation on $(\Omega, \mathcal{F}$, prob). It can be proved that

$$
E\left(\mathcal{N}_{p}((0, t] \times K)\right)=\lambda t \widetilde{n}(K)
$$

for $t \in(0, \infty), K \in \mathcal{G}$.

By a solution of (1), (2) we mean a stochastic process $(\xi(t))_{t \geq 0}$ with values in $\mathbb{R}^{d}$ such that with probability one the following two conditions are satisfied:

(a) The sample paths are right-continuous functions such that for $t>0$ the limit

$$
\xi(t-)=\lim _{s \rightarrow t-0} \xi(s)
$$

exists, and

(b) $\xi(t)=\xi_{0}+\int_{0}^{t} a(\xi(s)) d s+\int_{0}^{t} \int_{\Theta} \sigma(\xi(s-), \theta) \mathcal{N}_{p}(d s, d \theta) \quad$ for $t \geq 0$.

It is easy to write an explicit formula for the solution of (1), (2). Denote by $\pi^{t}$ the dynamical system defined by

$$
\pi^{t}(x)=y(t) \quad \text { for } t \in \mathbb{R}^{d},
$$

where $y$ is the solution of the ordinary differential equation

$$
y^{\prime}(t)=a(y(t)) \quad \text { for } t \in \mathbb{R}^{+},
$$


with the initial condition

$$
y(0)=x \text {. }
$$

Then for every fixed value of $p=\left(t_{i}, \theta_{i}\right)_{i \in \mathbb{N}}$ the solution of $(1),(2)$ is given by

$$
\begin{aligned}
\xi(0)=\xi_{0}, \quad \xi\left(t_{i}\right) & =\xi\left(t_{i}-\right)+\sigma\left(\xi\left(t_{i}-\right), \theta_{i}\right), \quad i \in \mathbb{N}, \\
\xi(t) & =\pi^{t-t_{i}}\left(\xi\left(t_{i}\right)\right) \quad \text { for } t \in\left[t_{i}, t_{i+1}\right), i \in \mathbb{N}_{0} .
\end{aligned}
$$

For $x \in \mathbb{R}^{d}$ denote by $\xi_{x}(t)$ the solution of the initial value problem (1), (2) with $\xi_{0}=x$. For every $t \geq 0$ and $f \in C_{\mathrm{b}}$ define

$$
U^{t} f(x)=E\left(f\left(\xi_{x}(t)\right)\right) .
$$

The classical theory of equation (1) ensures that under conditions (i)-(vi), $\left(\xi_{x}(t)\right)_{t \geq 0}$ is a homogeneous in time Markov process and $\left(U^{t}\right)_{t \geq 0}$ is a continuous semigroup of bounded linear operators acting on $C_{\mathrm{b}}$. Analogously for given $\mu \in \mathcal{M}_{1}$ we may find a solution $\xi_{\mu}(t), t \geq 0$, of (1), (2) such that $\xi_{\mu}(0)$ has distribution $\mu$. For every $t \geq 0$ we define $P^{t} \mu$ as the distribution of $\xi_{\mu}(t)$, i.e.

$$
P^{t} \mu(A)=\operatorname{prob}\left(\xi_{\mu}(t) \in A\right) \quad \text { for } t \geq 0, A \in \mathcal{B}_{\mathbb{R}^{d}} .
$$

The operators $P^{t}$ and $U^{t}$ satisfy the duality condition

$$
\left\langle f, P^{t} \mu\right\rangle=\left\langle U^{t} f, \mu\right\rangle \quad \text { for } t \geq 0, f \in C, \mu \in \mathcal{M}_{1} .
$$

Via (31) the semigroup $\left(P^{t}\right)_{t \geq 0}$ can be easily extended to the vector space $\mathcal{M}_{\text {sig }}$. It is locally Lipschitzian and weakly continuous. Moreover using the Phillips perturbation theorem it is easy to find an explicit formula for $\left(U^{t}\right)_{t \geq 0}$.

In fact, let $G_{0}$ be a linear operator given by the formula

$$
G_{0} f(x)=\int_{\Theta} f(q(x, \theta)) \widetilde{n}(d \theta) \quad \text { for } f \in C_{\mathrm{b}}\left(\mathbb{R}^{d}\right), x \in \mathbb{R}^{d},
$$

and let $\left(T^{t}\right)_{t \geq 0}$ be the semigroup corresponding to the unperturbed system $(27)$, i.e.:

$$
T^{t} f(x)=f\left(\pi^{t}(x)\right) \quad \text { for } f \in C_{\mathrm{b}}\left(\mathbb{R}^{d}\right), x \in \mathbb{R}^{d} .
$$

Then (cf. [12])

$$
U^{t} f=e^{-\lambda t} \sum_{n=0}^{\infty} U_{n}^{t} f \quad \text { for } f \in C_{\mathrm{b}}\left(\mathbb{R}^{d}\right),
$$

where

$$
\begin{aligned}
U_{n+1}^{t} f & =\lambda \int_{0}^{t} T^{t-s} G_{0} U_{n}^{s} f d s, \quad n=0,1, \ldots, \\
U_{0}^{t} f & =T^{t} f \quad \text { for } t \geq 0 .
\end{aligned}
$$


Using these formulas and Theorem 2 we can prove an explicit sufficient condition for the asymptotic stability of $\left(P^{t}\right)_{t \geq 0}$.

Theorem 3. Assume that assumptions (i)-(vi) are satisfied. Moreover, let $\lambda \geq 0$ and $\gamma \in \mathbb{R}$ be constants such that

$$
\left\|\pi^{t} x-\pi^{t} y\right\| \leq e^{\gamma t}\|x-y\| \quad \text { for } x, y \in \mathbb{R}^{d}, t \geq 0,
$$

and

$$
l_{q} \lambda+\gamma<\lambda
$$

Further, suppose that

$$
\langle a(x) \mid 2 x\rangle+\lambda \int_{\Theta}\left(\|x+\sigma(x, \Theta)\|^{2}-\|x\|^{2}\right) \widetilde{n}(d \theta) \leq \alpha_{0}\|x\|^{2}+\beta_{0}
$$

for some $\alpha_{0} \leq 0, \beta_{0} \in \mathbb{R}$. Then the semigroup $\left(P^{t}\right)_{t \geq 0}$ is asymptotically stable.

Proof. We show that $\left(U^{t}\right)_{t \geq 0}$ satisfies the assumptions of Theorem 2. We will verify by induction that for every $n=0,1, \ldots$ and $t \geq 0$,

$$
\left|\left(U_{n}^{t} f\right)(x)-\left(U_{n}^{t} f\right)(y)\right| \leq e^{\gamma t} \frac{\left(\lambda l_{q} t\right)^{n}}{n !}\|x-y\|, \quad x, y \in \mathbb{R}^{d} .
$$

Fix $f \in \mathcal{H}$ and $t \geq 0$. For $n=0$ by (33) and (35) we obtain

$$
\begin{aligned}
\left|\left(U_{0}^{t} f\right)(x)-\left(U_{0}^{t} f\right)(y)\right| & \leq\left|f\left(\pi^{t} x\right)-f\left(\pi^{t} y\right)\right| \leq\left\|\pi^{t} x-\pi^{t} y\right\| \\
& \leq e^{\gamma t}\|x-y\| \quad \text { for } x, y \in \mathbb{R}^{d} .
\end{aligned}
$$

From (32), (33) and (35) it follows immediately that

$$
\begin{aligned}
\mid\left(G_{0} U_{n}^{s} f\right)(x)- & \left(G_{0} U_{n}^{s} f\right)(y)\left|\leq \int_{\Theta}\right|\left(U_{n}^{s} f\right)(q(x, \theta))-\left(U_{n}^{s} f\right)(q(y, \theta)) \mid \widetilde{n}(d \theta) \\
& \leq e^{\gamma s} \frac{\left(\lambda l_{q} s\right)^{n}}{n !} \int_{\Theta}\|q(x, \theta)-q(y, \theta)\| \widetilde{n}(d \theta) \\
& \leq e^{\gamma s} l_{q}^{n+1} \frac{(\lambda s)^{n}}{n !}\|x-y\| \quad \text { for } x, y \in \mathbb{R}^{d} \text { and } s \in[0, t] .
\end{aligned}
$$

For $s \in[0, t]$ and $f \in \mathcal{H}$ we also have

$$
\begin{aligned}
\left|T^{t-s} G_{0} U_{n}^{s} f(x)-T^{t-s} G_{0} U_{n}^{s} f(y)\right| & \leq e^{\gamma(t-s)} e^{\gamma s} l_{q}^{n+1} \frac{(\lambda s)^{n}}{n !}\|x-y\| \\
& =e^{\gamma t} l_{q}^{n+1} \frac{(\lambda s)^{n}}{n !}\|x-y\| \quad \text { for } x, y \in \mathbb{R}^{d} .
\end{aligned}
$$

This and (35) imply (39). From (34) and (39) we obtain

$$
\left|U^{t} f(x)-U^{t} f(y)\right| \leq e^{\gamma-\lambda+l_{q} \lambda}\|x-y\|, \quad x, y \in \mathbb{R}^{d}, f \in \mathcal{H} .
$$

Since $\gamma-\lambda+l_{q} \lambda<0$, this implies condition (22) of Theorem 2. 
Now from the theory of semigroups, it follows that

$$
U^{t} f(x)=f(x)+\int_{0}^{t} U^{s} A_{U} f(x) d s \quad \text { for } x \in \mathbb{R}^{d}, f \in C_{\mathrm{c}}^{1}\left(\mathbb{R}^{d}\right),
$$

where

$$
A_{U} f(x)=\left\langle a(x) \mid f_{x}(x)\right\rangle-\lambda f(x)+\lambda \int_{\Theta} f(x+\sigma(x, \theta)) \widetilde{n}(d \theta) .
$$

Using this and an appropriate approximation we obtain

$$
U^{t} V(x)=V(x)+\int_{0}^{t} U^{s} \psi(x) d s \quad \text { for } x \in \mathbb{R}^{d},
$$

where $V(x)=\|x\|^{2}$ and

$$
\psi(x)=\langle a(x) \mid 2 x\rangle+\lambda \int_{\Theta}\left(\|x+\sigma(x, \theta)\|^{2}-\|x\|^{2}\right) \widetilde{n}(d \theta) .
$$

From (38), (43), (44) we deduce the inequality

$$
\frac{d U^{t}}{d t} \leq \alpha_{0} U^{t} V(x)+\beta_{0} .
$$

Solving (45) we conclude that

$$
U^{t} V(x) \leq V(x) e^{\alpha_{0} t}+\frac{\beta_{0}}{\alpha_{0}}\left(e^{\alpha_{0} t}-1\right) \quad \text { for } x \in \mathbb{R}, t \geq 0 .
$$

Since $\alpha_{0} \leq 0$ this implies (23). Thus by Theorem 2 the semigroup $\left(P^{t}\right)_{t \geq 0}$ is asymptotically stable.

The asymptotic properties of the semigroup $\left(P^{t}\right)_{t \geq 0}$ were studied by J. Traple [12] under different conditions. Namely, in [12] it was assumed that the dual semigroup $\left(U^{t}\right)_{t \geq 0}$ satisfies the equation

$$
u_{t}(t, x)=\left\langle a(x) \mid u_{x}(t, x)\right\rangle-\lambda(x) u(t, x)+\lambda(x) \int_{\Theta} u(t, x+\sigma(x, \theta)) \widetilde{n}(d \theta),
$$

where

$$
u(t, x)=U^{t} f(x), \quad u(0, x)=f(x) \quad \text { for } x \in \mathbb{R}^{d}, f \in C_{\mathrm{b}},
$$

and $\lambda: \mathbb{R}^{d} \rightarrow \mathbb{R}^{+}$is a function satisfying

$$
0<\underline{\lambda} \leq \lambda(x) \leq \bar{\lambda}<\infty, \quad|\lambda(x)-\lambda(y)| \leq|x-y| \quad \text { for } x, y \in \mathbb{R}^{d} .
$$

J. Traple proved the asymptotic stability of $\left(P^{t}\right)_{t \geq 0}$ if

and

$$
\langle a(x) \mid x\rangle+\lambda(x) \int_{\Theta}\langle\sigma(x, \Theta) \mid x\rangle \widetilde{n}(d \theta) \leq \alpha_{0}\|x\|^{2}+\beta_{0} \quad \text { for } x \in \mathbb{R}^{d},
$$

$$
2 \alpha_{0}<-\bar{\lambda} l_{\sigma}^{2}
$$

In our case $\lambda$ is a constant but we have replaced condition (47) by $\alpha_{0}<0$. 


\section{References}

[1] H. Gacki, An application of the Kantorovich-Rubinstein maximum principle in the stability theory of Markov operators, Bull. Polish Acad. Sci. Math. 46 (1998), 215223.

[2] J. Hutchinson, Fractals and self-similarity, Indiana Univ. Math. J. 30 (1981), 713747.

[3] N. Ikeda and S. Watanabe, Stochastic Differential Equations and Diffusion Processes, North-Holland, Amsterdam, 1981.

[4] L. V. Kantorovich and G. S. Rubinstein, On a space of completely additive functions, Vestnik Leningrad Univ. 13 (1958), 52-59 (in Russian).

[5] A. Lasota, Invariant principle for discrete time dynamical systems, Univ. Iagell. Acta Math. 31 (1994), 111-127.

[6] - From fractals to stochastic differential equations, in: Chaos-The Interplay Between Stochastic and Deterministic Behaviour (Karpacz, 1995), P. Garbaczewski et al. (eds.), Lecture Notes in Phys. 457, Springer, Berlin, 1995, 235-255.

[7] A. Lasota and M. C. Mackey, Chaos, Fractals, and Noise, Springer, Berlin, 1994.

[8] A. Lasota and J. Traple, An application of the Kantorovich-Rubinstein maximum principle in the theory of the Tjon-Wu equation, J. Differential Equations 159 (1999), 578-596.

[9] A. Lasota and J. A. Yorke, Lower bound technique for Markov operators and iterated function systems, Random Comp. Dyn. 2 (1994), 41-77.

[10] J. Malczak, Statistical stability of Poisson driven differential equations, Bull. Polish Acad. Sci. Math. 41 (1993), 159-176.

[11] S. T. Rachev, Probability Metrics and the Stability of Stochastic Models, Wiley, New York, 1991.

[12] J. Traple, Markov semigroups generated by Poisson driven differential equations, Bull. Polish Acad. Sci. Math. 44 (1996), 160-182.

[13] —, On the asymptotic stability of Markov semigroups, ibid. 44 (1996), 183-195.

\section{Henryk Gacki}

Institute of Mathematics

Silesian University

Bankowa 14

40-007 Katowice, Poland

E-mail: gacki@ux2.math.us.edu.pl 